\title{
Efficient Computation of Structural and Electronic Properties of Halide Perovskites Using Density Functional Tight Binding: GFN1- xTB Method
}

\author{
José Manuel Vicent-Luna, Sofia Apergi, and Shuxia Tao* \\ Cite This: J. Chem. Inf. Model. 2021, 61, 4415-4424 \\ Read Online
}

ABSTRACT: In recent years, metal halide perovskites (MHPs) for optoelectronic applications have attracted the attention of the scientific community due to their outstanding performance. The fundamental understanding of their physicochemical properties is essential for improving their efficiency and stability. Atomistic and molecular simulations have played an essential role in the description of the optoelectronic properties and dynamical behavior of MHPs, respectively. However, the complex interplay of the dynamical and optoelectronic properties in MHPs requires the simultaneous modeling of electrons and ions in relatively large systems, which entails a high computational cost, sometimes not affordable by the standard quantum mechanics methods, such as density functional theory (DFT). Here, we explore the suitability

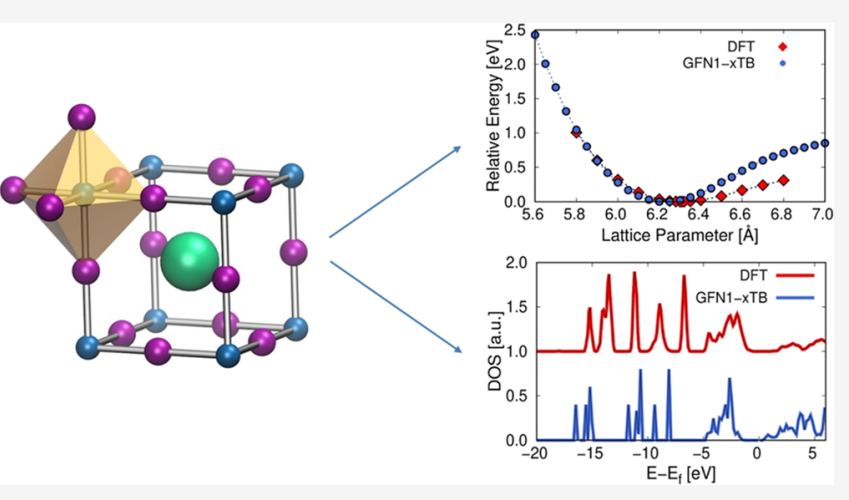
of the recently developed density functional tight binding method, GFN1-xTB, for simulating MHPs with the aim of exploring an efficient alternative to DFT. The performance of GFN1-xTB for computing structural, vibrational, and optoelectronic properties of several MHPs is benchmarked against experiments and DFT calculations. In general, this method produces accurate predictions for many of the properties of the studied MHPs, which are comparable to DFT and experiments. We also identify further challenges in the computation of specific geometries and chemical compositions. Nevertheless, we believe that the tunability of GFN1-xTB offers opportunities to resolve these issues and we propose specific strategies for the further refinement of the parameters, which will turn this method into a powerful computational tool for the study of MHPs and beyond.

\section{INTRODUCTION}

Metal halide perovskites (MHPs) are novel semiconductors that have gained great scientific attention in the recent years due to their excellent optoelectronic properties, which make them suitable for applications such as perovskite solar cells and light-emitting diodes. ${ }^{1-5}$ MHPs have the chemical formula $\mathrm{ABX}_{3}$, where $\mathrm{A}$ is a monovalent organic or inorganic cation $\left[\mathrm{Cs}^{+}, \mathrm{CH}_{3} \mathrm{NH}_{3}{ }^{+}\right.$, and $\left.\mathrm{CH}\left(\mathrm{NH}_{2}\right)_{2}{ }^{+}\right], \mathrm{B}$ is a metal divalent cation (typically $\mathrm{Pb}^{2+}$ or $\mathrm{Sn}^{2+}$ ), and $\mathrm{X}$ is halide anions $\left(\mathrm{I}^{-}, \mathrm{Br}^{-}\right.$, and, to a lesser extent, $\mathrm{Cl}^{-}$). Combining these compounds results in a semiconductor that exhibits suitable band gaps, high light absorption performance, low exciton binding energies, long carrier diffusion lengths, and high charge carrier mobility. ${ }^{6,7}$ In addition, MHPs exhibit a competitive fabrication cost together with a simple route to synthesize. Despite all these desirable properties, instability issues critically hamper their industrial application. ${ }^{8,9}$

Nowadays, many experimental and theoretical researchers are engaged in extending the understanding of the fundamental physicochemical properties of MHPs, which is crucial for increasing their stability. ${ }^{10-13}$ Computational modeling has proven to be a valuable tool to this endeavor since it can provide essential atomistic and microscopic insights into the fundamental properties of materials that are difficult, if not impossible, to obtain experimentally. ${ }^{12,14,15}$ There are diverse computational methods, and each has its own advantages and limitations to investigate processes at different sizes and time scales. Density functional theory (DFT) is the golden standard in materials science in predicting material properties. In recent years, DFT calculations have been used to study many properties of MHPs, such as geometrical, ${ }^{16,17}$ optoelectronic, ${ }^{16-18}$ and vibrational properties, ${ }^{19}$ enthalpies of formation, ${ }^{20}$ defect activity, ${ }^{21}$ and ion migration, ${ }^{22}$ among others. On the other hand, molecular dynamics simulations based on classical force fields have proven to be useful in the study of the dynamical features of MHPs, such as ionic

Received: April 21, 2021

Published: August 20, 2021 
diffusion, ${ }^{23,24}$ structural phase transitions, ${ }^{24,25}$ thermal and ionic conductivities, ${ }^{24}$ or phonon density of states (DOS). ${ }^{26}$

Despite the advantages of the aforementioned techniques, there are also plenty of limitations. The high computational cost of DFT calculations limits the study to small systems and short timescales. This is a considerable restriction since many of the most relevant and challenging advances in MHPs require the study of larger systems. These include alloys combining several cations, metals, and anions, ${ }^{27}$ the effect of the concentration of vacancies and defects in the crystal, ${ }^{28}$ the confinement of MHPs within porous materials such as silica matrices $^{29}$ or metal-organic frameworks, ${ }^{30}$ and the interface of perovskites with other materials acting as charge-transport layers. $^{31,32}$ Classical simulations seem to be an "in part" solution to the above-mentioned size limitations of DFT calculations; however, they suffer from other drawbacks, such as the inability to simulate electrons and chemical reactions that are essential for the description of many properties of MHPs. Besides, classical simulations need a suitable and realistic force field, which is challenging to parameterize.

Semiempirical quantum mechanics methods, such as density functional tight binding (DFTB), could provide an ideal and complementary platform, combining the functionalities of both electronic and ionic description. ${ }^{33}$ Traditional DFTB methods are based on simplifying the Kohn-Sham DFT total energy as a function of the electron density using precomputed interactions of element pairs, considerably reducing the computational cost. ${ }^{34}$ These pair interactions as a function of the distance are tabulated and stored in the so-called SlaterKoster files. However, this parameterization lacks transferability and is limited to a number of elements. Parameters for the most common perovskite constituents, such as $\mathrm{Cs}, \mathrm{Pb}$, $\mathrm{Sn}$, and the halides, are not yet available.

GFN1-xTB is a new extended tight binding method, recently developed by Grimme et al., that covers most of the elements of the periodic table up to $Z=86 .^{35}$ To the best of our knowledge, GFN1-xTB is the first DFTB method that includes a complete parameterization of all the atoms existing in MHPs. This method comprises a limited number of physically interpretable parameters that can be refined to study several key properties of given material systems. The GFN1-xTB method was first designed for the calculation of molecular complexes but not for periodic systems. ${ }^{35}$ Recently, the computation of periodic systems became possible in the Amsterdam density functional suite; ${ }^{36}$ however, its performance is still unknown.

In this work, we investigate the effectiveness of GFN1-xTB by calculating the main properties of MHPs via a comprehensive comparison with DFT and experimental data. To achieve that, we analyze the energetic, structural, electronic, and vibrational properties of all primary (18) MHPs with the formula $\mathrm{ABX}_{3}\left(\mathrm{~A}=\mathrm{CH}_{3} \mathrm{NH}_{3}{ }^{+}\right.$or $\mathrm{MA}^{+}, \mathrm{CH}\left(\mathrm{NH}_{2}\right)_{2}{ }^{+}$or $\mathrm{FA}^{+}$, and $\mathrm{Cs}^{+} ; \mathrm{B}=\mathrm{Pb}^{2+}$ and $\mathrm{Sn}^{2+}$; and $\mathrm{X}=\mathrm{I}^{-}, \mathrm{Br}^{-}$, and $\mathrm{Cl}$ ) in their cubic, tetragonal, and orthorhombic forms. Our results suggest that the original parameterization of GFN1-xTB describes targeted properties of MHPs properly. However, its performance in geometry relaxation calculations still requires further improvements, especially for the structures with a lower symmetry. We find that electronic properties of formamidinium cations are incorrectly predicted due to the presence of complex chemical bonds, such as dynamic covalent bonds. We conclude that GFN1-xTB is a promising method for the study of molecular and periodic systems of larger sizes, unattainable for standard DFT. However, further targeted refinement of its parameters is required to eliminate the current limitations.

\section{SIMULATION DETAILS}

The DFTB simulations presented in this work were carried out in AMS2019.3 SCM software, ${ }^{36}$ with the implementation of the GFN1-xTB method ${ }^{35}$ for periodic systems. The GFN1xTB Hamiltonian comprises four independent terms based on functional forms with adjustable parameters: electronic, repulsion, dispersion, and halogen-bonding terms (see refs 35 and 37 for a detailed description of the method). The electronic contribution to the energy is the most relevant term of this tight binding method, which takes the electronic structure, the electrostatic, and the exchange-correlation energy into account. The repulsion energy is approximated by a classical expression that is independent of the electronic term. The repulsion term is intended to correct the changes in the short-range interactions originated by the overlap of the atomic reference densities. ${ }^{37}$ The third term, that is, the dispersion energy, takes into account the long-range correlation effects because of the London dispersion interactions. Here, the dispersion energy is computed by the D3 method ${ }^{38}$ using the BJ-damping scheme ${ }^{39}$ without threebody terms and adjusted short-range damping parameters for the GFN1-xTB method. ${ }^{35}$ Finally, the halogen-bonding term is included as a repulsive correction for the deficiencies in the description of the halogen bonds. It is worth mentioning that MHPs have a complex potential energy surface (PES) because they can be stable in different structural phases. In order to simplify the PES, in this work, we do not use the halogen bond contribution to the energy. This is justified because it is a minor correction to the energy, but it can lead to a noncontinuous PES, which is not desirable for the geometry optimization calculations of periodic systems. ${ }^{36}$ It should be noted that in this work, we have not modified the parameters of the GFN1-xTB Hamiltonian.

We used the fast inertial relaxation engine ${ }^{40}$ (FIRE) optimizer to perform all the geometry relaxations using the GFN1-xTB method. The nuclear gradient convergence and the energy threshold for the stress tensor when optimizing lattice vectors were set to 0.001 Hartree/ $\AA$ and 0.005 Hartree, respectively. Note that the FIRE optimizer does not use an energy criterion convergence, but the convergence relies on changes on forces (nuclear gradients) and stress tensor. The threshold to determine the radius of the basis functions was fixed to 0.0001, and the Coulombic interactions were computed with the Ewald summation method with a tolerance of $10^{-8}$. We used a regular grid for the $k$-space integration $(k$ points) based on a quadratic interpolation method. ${ }^{36}$ The number of $k$-points is analyzed in the first part of the Results and Discussion section. The initial structures of each MHP in its different phases were taken from the DFT-optimized structures in a previous work of Tao et al. ${ }^{16}$

DFT calculations were performed using the projector augmented-wave method as implemented in the Vienna $\mathrm{Ab}$ initio Simulation Package (VASP). ${ }^{41-44}$ The electronic exchange-correlation interaction was described by the functional of Perdew, Burke, and Ernzerhof (PBE) within the generalized gradient approximation. ${ }^{45}$ Energy and force convergence criteria of $10^{-5} \mathrm{eV}$ and $2 \times 10^{-2} \mathrm{eV} / \AA$, respectively, were used in all calculations, along with a kinetic energy cutoff of $500 \mathrm{eV}$ and a $4 \times 4 \times 4 k$-point grid. The D3 correction that accounts for the van der Waals interactions was 
(a)

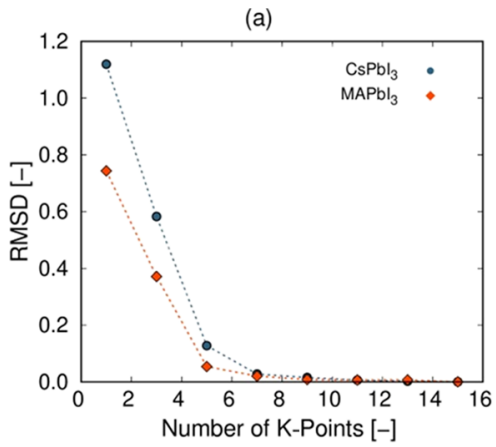

(b)

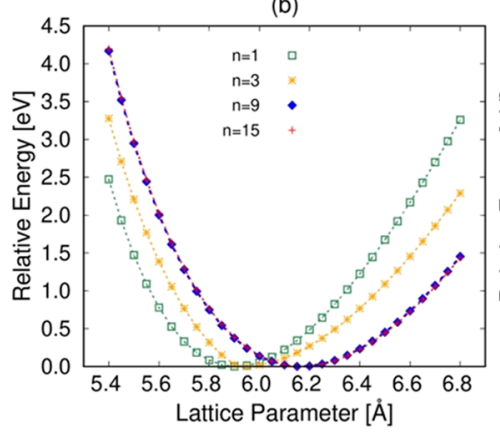

(c)

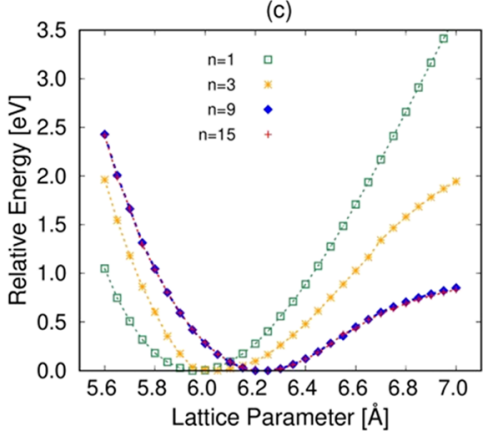

Figure 1. rmsd as a function of the number of $k$-points with $n=15$ as the reference (a) and $k$-point dependence of the energy of GFN1-xTBoptimized cubic $\mathrm{CsPb}_{3}$ (b) and $\mathrm{MAPbI}_{3}$ (c) as a function of the lattice parameter. The energy of the optimal structure is set to zero. For clarity, only a set of representative $k$-points are presented (see Figure S1 for the complete set). $n$ stands for $(n \times n \times n) k$-points in the three directions.
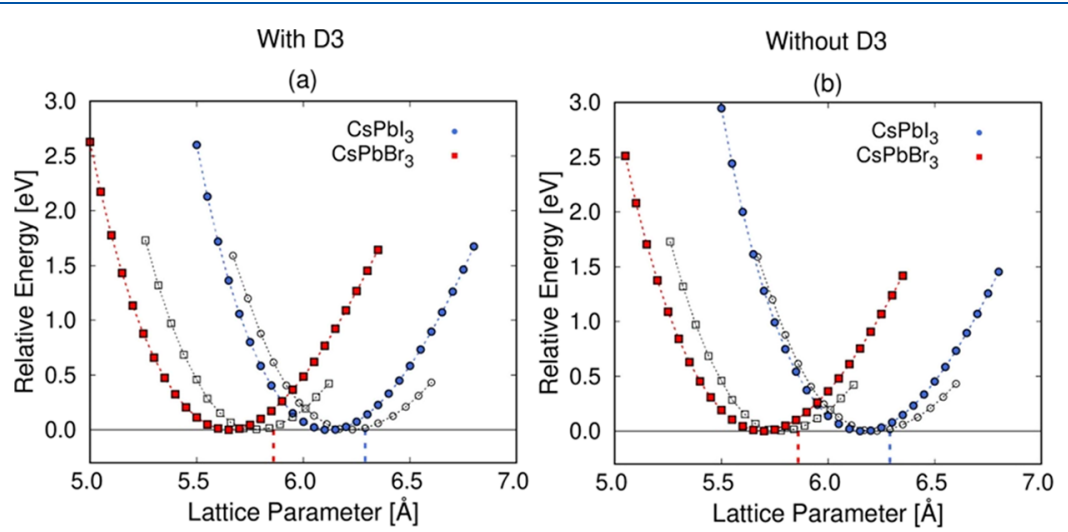

(c)
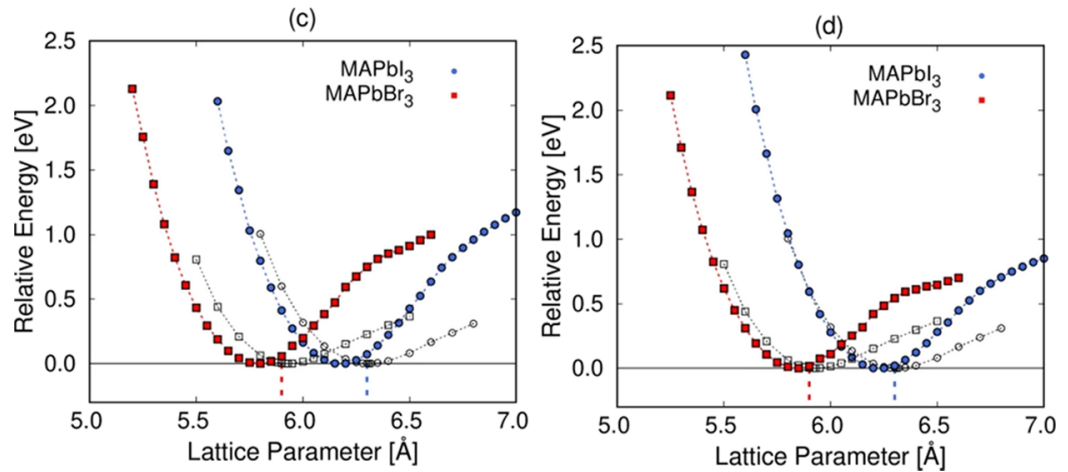

Figure 2. Relative GFN1-xTB energy as a function of the lattice parameter for the cubic $\mathrm{CsPbI} / \mathrm{MAPbI}_{3}$ (blue circles) and $\mathrm{CsPbBr} 3 / \mathrm{MAPbBr} 3$ (red squares), with (a)/(c) and without D3 (b)/(d) dispersion corrections. DFT data (clear symbols) using the PBE + D3 functional are included for comparison. The vertical dashed lines represent the experimental lattice parameters for each MHP. ${ }^{51-54}$

employed when specified. ${ }^{38}$ In addition, reference DFT data using the PBEsol functional used for comparison were taken from a previous publication of Tao et al. ${ }^{16}$

\section{RESULTS AND DISCUSSION}

Structural Properties. K-Points Convergence. The number of $k$-points used in quantum calculations to sample the Brillouin zone is an important parameter that influences the accuracy of the results. The use of many $k$-points not only ensures higher precision but also increases the computational cost of the simulations. A compromise between accuracy and computational cost is necessary. We first performed a set of calculations to determine how the number of $k$-points affects the results. By performing small deformations, that is, isotropic expansions and compressions of the unit cell, we calculated the energy of the systems as a function of the lattice parameter.
The systems were confined in a fixed volume, and only the ionic positions were optimized, as described in the Simulation Details section. We selected cubic $\mathrm{CsPbI}_{3}$ and $\mathrm{MAPbI}_{3}$ as test systems with inorganic and organic cations, respectively. We analyzed the convergence of the $k$-points with $n=15 k$-points in each direction as the reference (where the total number of $k$ points is $n \times n \times n)$, a choice justified by our results, since the deviation of the computed energies compared with those obtained for $n=11$ and 13 is almost negligible (Figure S1).

Figure 1a shows the root-mean-squared deviation (rmsd) of the energies (Figure S1) with respect to the reference value ( $n$ $=15)$ as a function of the number of $k$-points, while Figure $1 b, c$ shows the energies of $\mathrm{CsPbI}_{3}$ and $\mathrm{MAPbI}_{3}$ MHPs as a function of the lattice parameter for a number of selected $k$ points. The number of $k$-points, as expected, affects not only the calculated minimum of the energy curve but also its shape. 

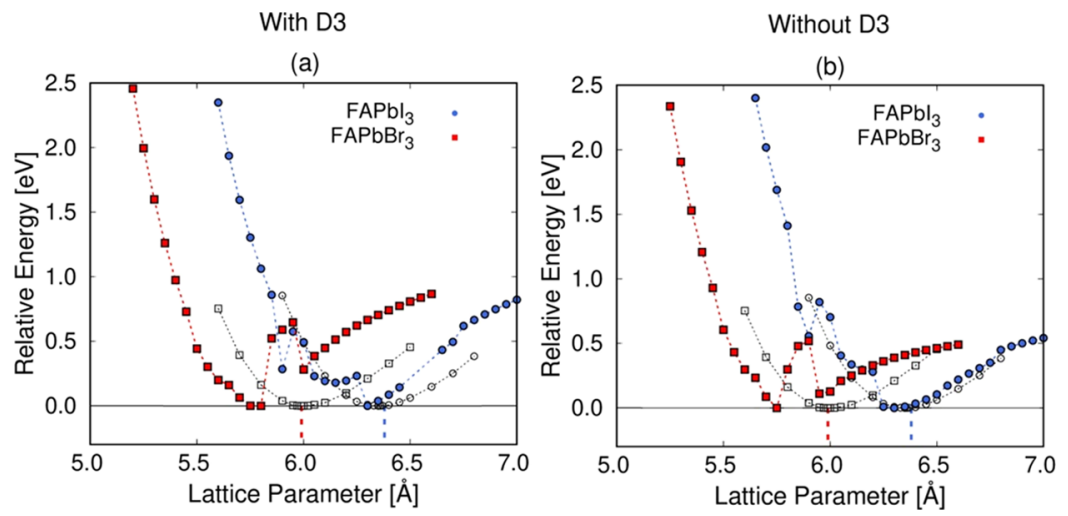

Figure 3. Relative energy as a function of the lattice parameter for the cubic $\mathrm{FAPbI}_{3}$ (blue circles) and $\mathrm{FAPbBr}_{3}$ (red squares) from GFN1-xTB with (a) and without D3 (b) dispersion corrections. DFT data (clear symbols) using the PBE + D3 functional are included for comparison. The vertical dashed lines represent the experimental lattice parameters for each MHP. ${ }^{16,55}$

Lower values of $\mathrm{n}$ lead to the prediction of smaller structures and more significant deviations on the extremes of the curves. The rmsd presented in Figure 1a decreases fast up to $n=5$ and plateaus for higher values. We found a good compromise between accuracy and computational cost for $n=9$, and we therefore chose this number of $k$-points to simulate systems with lattice parameters around $6 \AA$, which is the standard size of the unit cell of cubic perovskites. For the larger tetragonal and orthorhombic unit cells, we reduced the number of $k$ points accordingly; it is $7 k$-points for lattice parameters around $9 \AA$ and $5 k$-points for lattice parameters around $12 \AA$.

Equation of States. The ability of a computational method to describe the energy changes upon a small deformation of the structures around the equilibrium is important not only for the description of the materials but also for the development of potential parameters for classical simulations. ${ }^{23,25}$ To assess the ability of GFN1-xTB in this regard, we compared its performance with DFT (PBE + D3) in producing energy curves after isotropic distortions of the cubic MHPs. We also analyzed the effect of the dispersion correction in the simulation of MHP crystals.

In Figure 2, the results for lead-based MHPs containing $\mathrm{Cs}^{+}$ or $\mathrm{MA}^{+}$cations and $\mathrm{I}^{-}$and $\mathrm{Br}^{-}$anions are presented, with and without the dispersion energy (see the Simulation Details section for more details) and compared to DFT (PBE + D3) data. The D3 dispersion term tends to shift the curve to lower lattice parameters, resulting in the over-compression of the crystal. This correction describes the attractive part of the van der Waals interactions, which is very prominent in the molecular systems for which GFN1-xTB was initially developed to describe. In our case, the correction does not accurately describe the dispersion forces in the MHP crystals, therefore eliminating it from the GFN1-xTB Hamiltonian results in better agreement with the reference DFT (PBE + D3) data and experimental results. In line with this finding, previous studies have demonstrated that the use of D3 dispersion corrections often induces an over-compression of the crystals. ${ }^{46-50}$ These studies remark that a scaling of the dispersion terms is needed to describe the London dispersion interactions of some ionic crystals, ${ }^{46,50}$ while it is not sufficient in other cases where other functionals developed for the solidstate materials (such as PBEsol, PBEsol0, or wB97X) perform better. $^{47-49}$ In general, the DFTB relative energies are in good agreement with DFT (PBE + D3) for MHPs containing inorganic $\mathrm{Cs}^{+}$cations (Figure 2b). However, for $\mathrm{MA}^{+}-$ containing MHPs and for lattice parameters larger than the optimal, the calculated energies are slightly overestimated (Figure $2 \mathrm{~d}$ ). This means that the equation of state computed with GFN1-xTB is more attractive than the reference DFT data when the system is expanded over the equilibrium configuration.

DFT results are not unique but instead vary depending on the selected functional and/or calculation settings. Figure S2, for instance, compares the DFT lattice energies computed with the PBEsol and PBE functionals with and without the D3 dispersion term, and Table $\mathrm{S} 1$ collects the corresponding equilibrium lattice constants. We can see that the difference between the DFT with different functionals and GFN1-xTBcalculated relative energies is in the same range as the energy difference between two DFT functionals. We can therefore conclude that GFN1-xTB is suitable for the description of the relative lattice energies of these four selected MHPs. To complete the set of MHPs, Figures S3 and S4 show the relative energy data for the $\mathrm{CsPbCl}_{3}$ and $\mathrm{MAPbCl}_{3} \mathrm{MHPs}$ and the same set of Sn-based MHPs. The collective results show that the GFN1-xTB method correctly predicts the order of the equilibrium lattice parameters following the halide order: $\mathrm{Cl}^{-}<$ $\mathrm{Br}^{-}<\mathrm{I}^{-16}$ Regarding the metal, Pb-based MHPs exhibit slightly larger unit cells than the corresponding Sn-based MHPs, as expected. ${ }^{16}$

Out of all the studied compositions, the largest discrepancy is found in the $\mathrm{FA}^{+}$perovskites. Because $\mathrm{MA}^{+}$and $\mathrm{FA}^{+}$cations are quite similar, being formed by carbon, nitrogen, and hydrogen atoms, one would expect GFN1-xTB to perform similarly with structures containing these cations. However, as shown in Figure 3, upon compression and expansion of the unit cell, the relative energies of $\mathrm{FAPbI}_{3}$ and $\mathrm{FAPbBr}_{3}$ exhibit an erratic behavior. Specifically, we found that small changes in the lattice parameters of the perovskite produce relatively high energy jumps, contrary to the smooth trends depicted in Figure 2. This unexpected behavior is due to the molecular structure of the $\mathrm{FA}^{+}$cations. Unlike $\mathrm{MA}^{+}, \mathrm{FA}^{+}$cations contain a dynamic double bond between the carbon atom and one of the two attached nitrogen atoms. The presence of a double bond in a charged molecule affects the electronic configuration of the atoms, in a way that does not seem to be accounted for in the original GFN1-xTB parameterization, and as a result, the method fails to describe $\mathrm{FA}^{+}$-containing MHPs.

Organic Cation Rotation Barrier. Another significant property that a computational method should be able to 

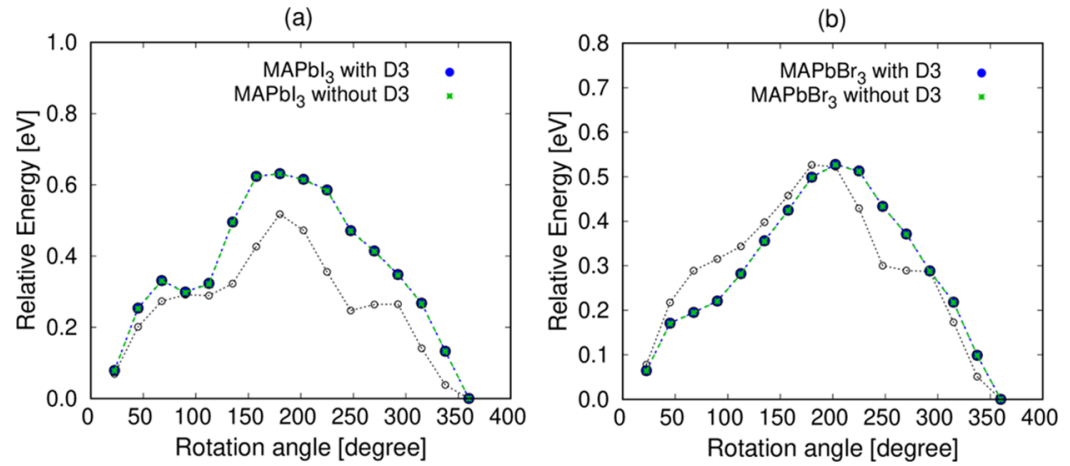

Figure 4. Relative GFN1-xTB energy as a function of the rotation angle of the organic $\mathrm{MA}^{+}$cations in the cubic $\mathrm{MAPbI}_{3}(\mathrm{a})$ and $\mathrm{MAPbBr}_{3}(\mathrm{~b})$ with D3 and without D3 dispersion corrections. DFT data (open symbols) using the PBE + D3 functional are included for comparison.

describe accurately is the configuration of the cations within the $\mathrm{PbI}_{6}$ octahedra of the MHPs. We put GFN1-xTB to the test by calculating the energy of our systems as a function of the $\mathrm{MA}^{+}$cation rotation angle. Specifically, starting from the equilibrium configuration, we rotated the $\mathrm{MA}^{+}$cations in the unit cells of $\mathrm{MAPbI}_{3}$ and $\mathrm{MAPbBr}_{3}$ around the $\mathrm{C}-\mathrm{N}$ axis and the energies were acquired by single-point calculations (Figure 4). We found good agreement between GFN1-xTB and DFT, with GFN1-xTB being able to reproduce the energy barrier for the rotation of $\mathrm{MA}^{+}$cations predicted with DFT $(\mathrm{PBE}+\mathrm{D} 3)$. Both methods suggest that the peak of the rotation energy barrier is around $180^{\circ}$ from the equilibrium angle. We observe slight differences in the energy values, which can be attributed to the fact that equilibrium geometries from GFN1-xTB and $\mathrm{DFT}(\mathrm{PBE}+\mathrm{D} 3)$ are slightly different, and the rotation energies can only be calculated via single-point calculations. It is also worth noting that for single-point calculations, the D3relative energies are almost the same (each configuration deviates less than $0.002 \mathrm{eV}$ ) as the ones without D3. This is because the geometry of the system does not change during the calculation, and then, the D3 term only contributes to the total energy with a constant value. Figure S5 shows the corresponding results for the rotation of $\mathrm{FA}^{+}$cations in $\mathrm{FAPbI}_{3}$, where the inability of GNF1-xTB to properly describe $\mathrm{FA}^{+}$is manifested once more.

Structural Optimization. All the previous results of relative energies are based on the calculations of the cubic systems having fixed lattice constants (volume) describing an equation of state. However, a successful computational method needs to be able to predict equilibrium structures through full geometry optimizations. In Figure 5, the results of the full geometry optimizations of the cubic, tetragonal, and orthorhombic phases of all MHPs studied in this work are compared with the DFT (PBEsol) reference values. In general, GFN1-xTB tends to underestimate the lattice parameters of the MHPs, resulting in an over-compression of the material (Figure 5a). Most of the GFN1-xTB-calculated data nevertheless follow the same trend as the reference. However, a few points deviate considerably from the reference values, indicating a vast deformation of some of the crystal structures.

To distinguish which structures and/or elements are harder for GFN1-xTB to describe, we analyzed the interatomic deviations for each system separately. The data are taken from Figure $5 b$, where the range of interatomic distances is chosen to take into account the relative positions between each atom of one unit cell with atoms of the adjacent unit cell. Figure 6 shows the percentage of deviation of the interatomic distances
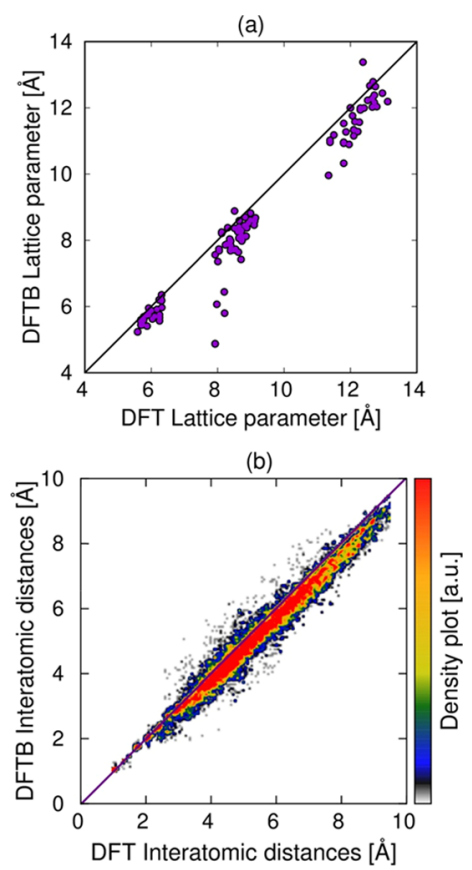

Figure 5. Comparison between computed DFT and GFN1-xTB (DFTB) lattice parameters (a) and interatomic distances (b) between all atom pairs in cubic, tetragonal, and orthorhombic MHPs. The reference DFT results were computed using the PBEsol functional without D3 dispersion corrections. ${ }^{16}$

by varying the $\mathrm{A}, \mathrm{B}$, and $\mathrm{X}$ species and the crystal shape. In general, GFN1-xTB predicts better the geometries of more symmetrical phases than the crystals with a less symmetry following the order: cubic $>$ tetragonal $>$ orthorhombic. The higher distortion is observed for orthorhombic phases reaching values of deviation up to $20-30 \%$ from the reference data, while cubic structures are predicted with a maximum error lower than $10-15 \%$.

MHPs containing $\mathrm{Cs}^{+}$and $\mathrm{I}^{-}$seem to be better predicted, and $\mathrm{Pb}$-based MHPs are better than their respective $\mathrm{Sn}$-based MHPs. Overall, the geometry seems to be a more influential factor than the composition. The method describes the simplest and high-symmetry cubic geometries better than the more complex and distorted orthorhombic phases. This can be related to the fact that the GFN1-xTB Hamiltonian is based on the interatomic distances between pairs of atoms, which are more uniformly distributed in more symmetrical systems. 

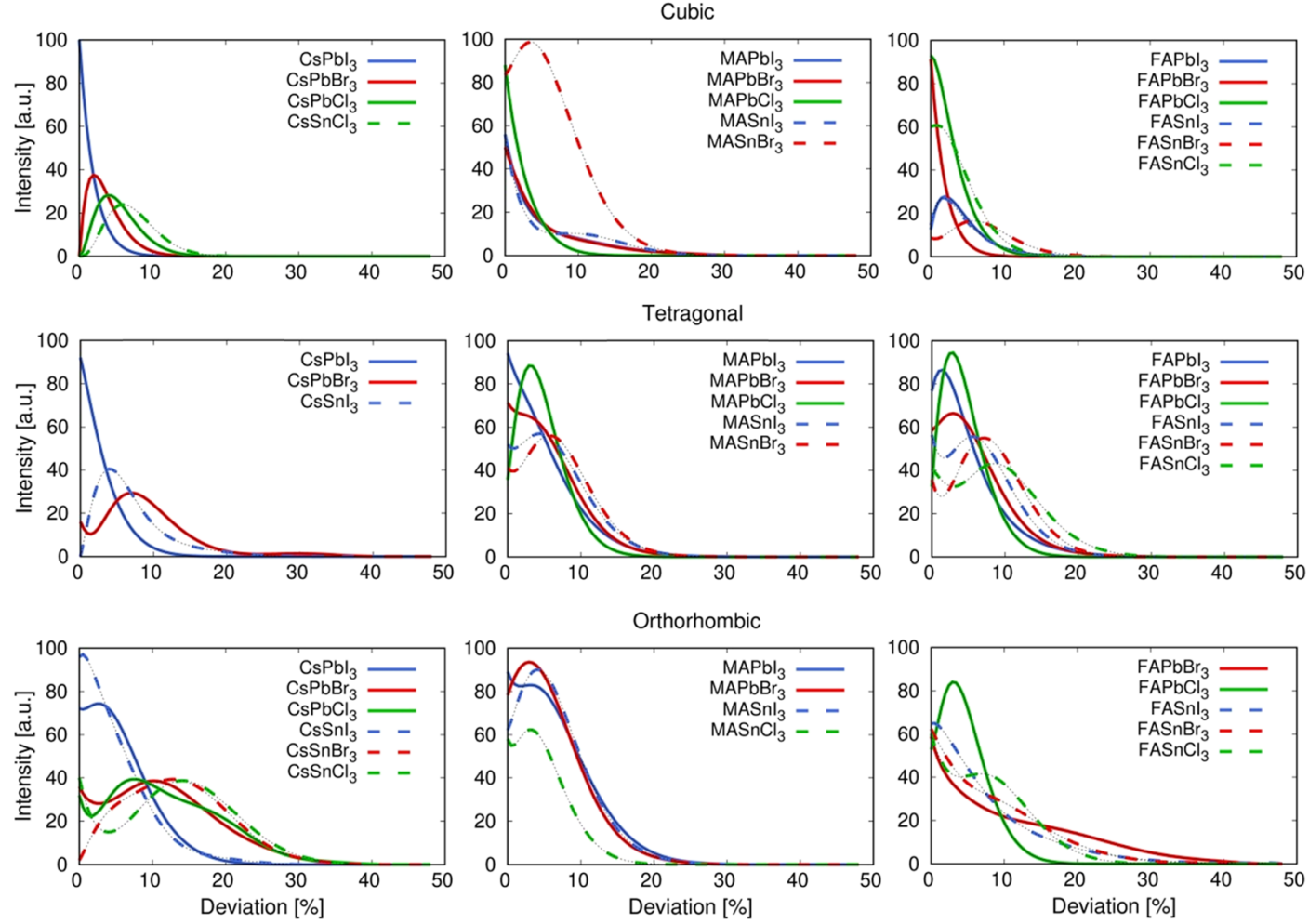

Figure 6. Histogram of the percentage of deviation of interatomic distances between all atom pairs calculated with GFN1-xTB with respect to the reference DFT values in cubic, tetragonal, and orthorhombic MHPs. The reference DFT results were computed using the PBEsol functional without D3 dispersion corrections. ${ }^{16}$

Electronic Properties. Band Structure. Another advantage of the DFTB methods over classical simulations is the ability to describe electronic properties. To benchmark the performance of GFN1-xTB in the prediction of the electronic properties of MHPs, we compared the GFN1-xTB-calculated electronic band structure of the most typical $\mathrm{CsPbI}_{3}$ and $\mathrm{MAPbI}_{3}$ MHPs with the respective DFT (PBE + D3) band structures. As can be seen in Figure 7, there is excellent agreement between GFN1-xTB and DFT, with the same observed trends for the more important bands, that is, those closer to the conduction and the valence bands. Both MHPs exhibit a direct band gap at the $\Gamma$ point with values of 1.91 and $1.93 \mathrm{eV}$ for $\mathrm{CsPbI}_{3}$ and 1.61 and $1.66 \mathrm{eV} \mathrm{MAPbI}$ obtained with DFT (PBE + D3) and GFN1-xTB calculations, respectively. We also confirmed that these predictions are in line with the experimental observations (Figure 8) by calculating the band gaps of the $\mathrm{CsBX}_{3}$ and $\mathrm{MABX}_{3}$ MHPs.

From the geometrical analysis, we know that some of the GFN1-xTB-optimized systems can suffer a considerable structural distortion. To account for these deformations and their effect on the calculated band gaps, we compared the band structures of the systems previously optimized with DFT (PBE + D3) (DFTB-DFT-opt) to those optimized with GFN1-xTB (DFTB-DFTB-opt). The results are presented in Figure 8, together with the experimental data reported by Tao et al. ${ }^{16}$ Most of the calculated band gaps are very close to the experiments and only a few deviate, with the largest differences observed for the $\mathrm{CsPbCl}_{3}$ and $\mathrm{CsSnCl}_{3}$ MHPs. GFN1-xTB also predicts the correct behavior of the band gap evolution when changing the halide anion, that is, the band gap increases as the size of the anion decreases. We can also observe that the GFN1-xTB optimization worsens the agreement with experi-

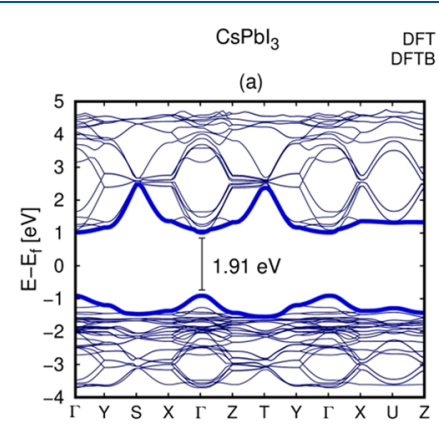

DFT $=\mathrm{MAPbl}_{3}$
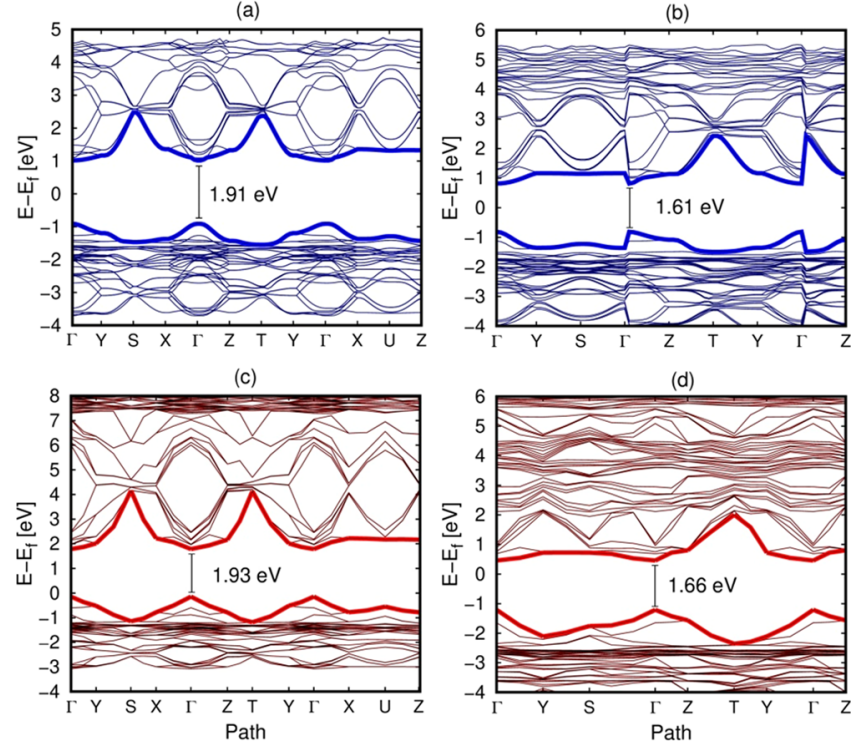

Figure 7. Calculated PBE + D3 (DFT, blue) and GFN1-xTB (DFTB, red) band structure for the orthorhombic $\mathrm{CsPbI}_{3}(\mathrm{a}, \mathrm{c})$ and tetragonal $\mathrm{MAPbI}_{3}(\mathrm{~b}, \mathrm{~d})$.

ments but still predicts the correct tendency. It is worth mentioning that GFN1-xTB can predict the band gaps of the perovskites similar to more expensive DFT calculations reported in the literature. ${ }^{18}$ We note that standard DFT generally underestimates band gaps; however, not taking into account spin orbit coupling/relativistic effects also leads to band gap predictions comparable to the experiments due to 


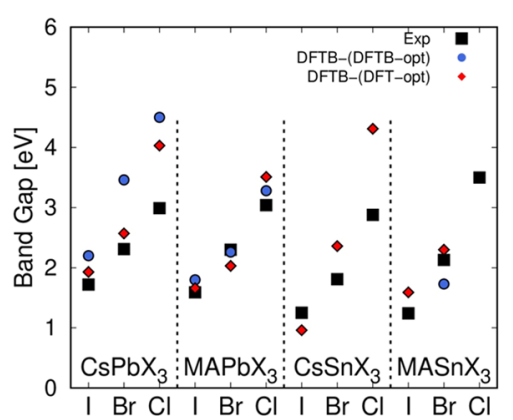

Figure 8. Comparison between experimental (black squares) and computed GFN1-xTB (DFTB) band gaps for the orthorhombic $\mathrm{CsBX}_{3}$ and tetragonal $\mathrm{MABX}_{3}(\mathrm{~B}=\mathrm{Pb}$ and $\mathrm{Sn}$ and $\mathrm{X}=\mathrm{I}, \mathrm{Br}$, and $\mathrm{Cl})$. The structures were previously optimized with GFN1-xTB (DFTB, blue circles) and PBEsol (DFT, red diamonds). Experimental values are taken from Tao et al. ${ }^{16}$

error cancelation. Nevertheless, our results indicate that GFN1-xTB is also suitable for the reasonable prediction of the electronic properties of MHPs.

Density of States. To analyze the electronic properties of the MHPs in more detail, we computed the contribution of each species to the electronic DOS. In Figure 9, the GFN1xTB-calculated partial DOS for the DFT (PBEsol)-optimized $\mathrm{CsPbI}_{3}, \mathrm{MAPbI}_{3}$, and $\mathrm{FAPbI}_{3}$ is compared to its DFT counterpart. In general, GFN1-xTB gives similar results to DFT (PBEsol), at least around the band gap; there are however some discrepancies far away from the band edges, with some peaks deviating up to $2 \mathrm{eV}$. The performance of GFN1-xTB is generally acceptable but with two notable exceptions being the absence of peaks for $\mathrm{Cs}^{+}$around -9 and $-14 \mathrm{eV}$ in $\mathrm{CsPbI}_{3}$ and a systematic shift to higher energies of the $\mathrm{FA}^{+}$signals. The latter deviation produces a peak within the band gap of $\mathrm{FAPbI}_{3}$, close to the valence band maximum that hinders the estimation of a reliable band gap value. In MHPs, there should not be any contribution of the cations between the valence and conduction bands. However, $\mathrm{MA}^{+}$ cations (which have the same elements than $\mathrm{FA}^{+}$cations) do not show any feature within the band gap. We attribute this to the presence of nonlocalized double $\mathrm{C}-\mathrm{N}$ bonds in $\mathrm{FA}^{+}$ cations. The presence of these complex bonds affect the electronic environment of $\mathrm{C}$ and $\mathrm{N}$ atoms, and it is not correctly captured by the GFN1-xTB method. The problem with the description of the electronic behavior of $\mathrm{FA}^{+}$seems to be the underlying reason for the erroneous description of the relative energies depicted in Figure 3.

Figure S6 shows the DOS before and after the structures have been optimized with the GFN1-xTB method. We can see that the structural changes caused by the full geometry optimization (Figures 5 and 6) do not significantly influence the general behavior of the electronic DOS; however, small energy displacements of the DOS peaks can be observed. These shifts are responsible for the differences in the computed band gaps depicted in Figure 8. It is worth mentioning that a proper optimization with GFN1-xTB before computing the electronic properties does not solve the incorrect description of the $\mathrm{DOS}$ of $\mathrm{FAPbI}_{3}$.

Vibrational Properties. The last examination conducted in this work involves the calculation of the vibrational properties of MHPs, which are a fundamental aspect for the study of the MHP stability and the differences between the various structural phases. ${ }^{19,51}$ In Figure 10, the GFN1-xTBcalculated phonon dispersion for the cubic $\mathrm{CsPbI}_{3}$ and $\mathrm{CsPbBr}_{3}$ is presented and compared to its DFT counterpart reported in the literature. ${ }^{56,57} \mathrm{~A}$ certain agreement is observed between the two methods, with most of the identified vibrational modes in both MHPs fluctuating around the same energies (or frequencies). Remarkably, GFN1-xTB predicts the existence of imaginary acoustic modes with negative energies or frequencies, also known as soft modes, at the $\mathrm{M}$ and $\mathrm{R}$ points, which is a common aspect of the cubic MHPs, indicative of the dynamical instabilities of the structures. Similarly, GFN1-xTB predicts the disappearance of the imaginary modes, when replacing the cubic with the
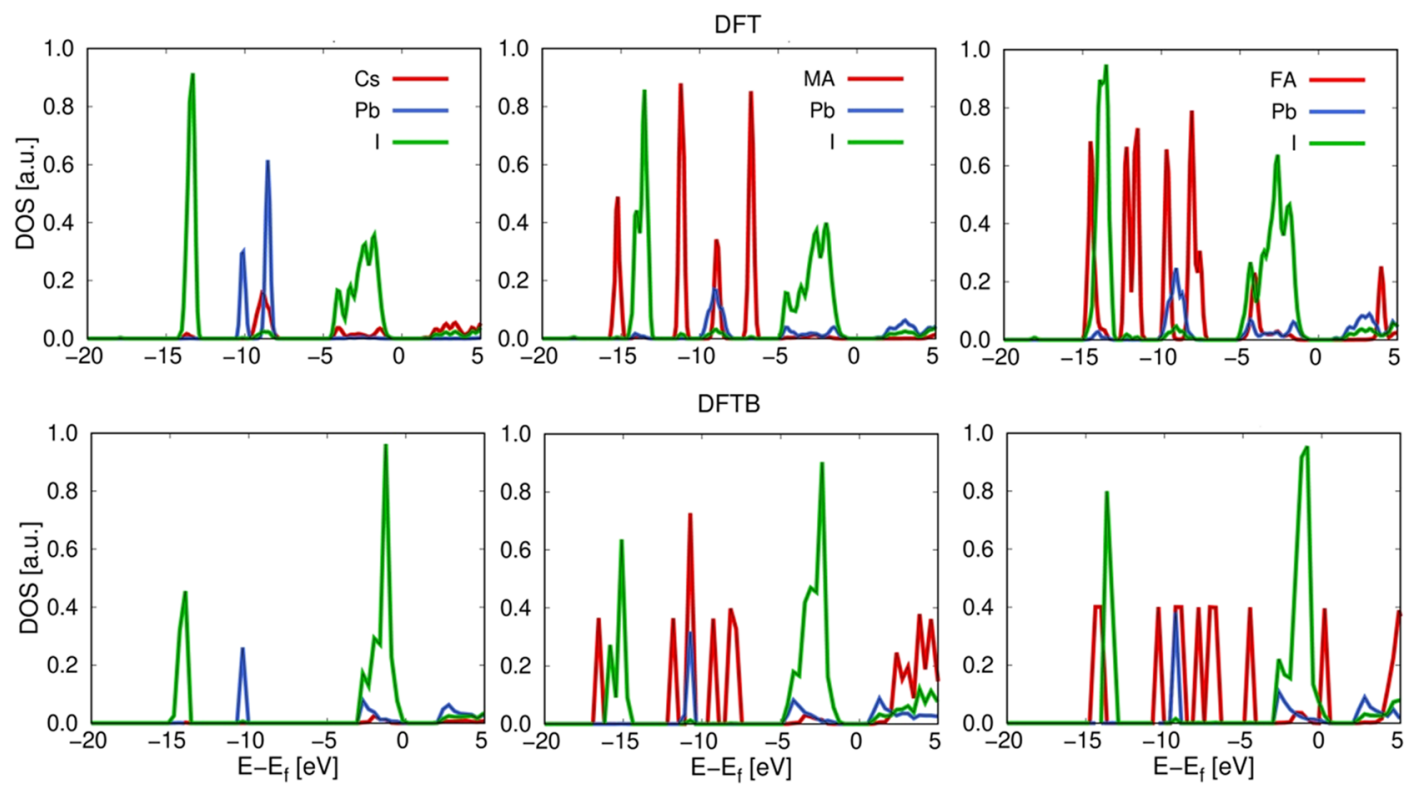

Figure 9. Partial DOS for the orthorhombic $\mathrm{CsPbI}_{3}$ and tetragonal $\mathrm{MAPbI}_{3}$ and $\mathrm{FAPbI}_{3}$ computed with PBEsol (DFT, top) and GFN1-xTB (DFTB, bottom). 


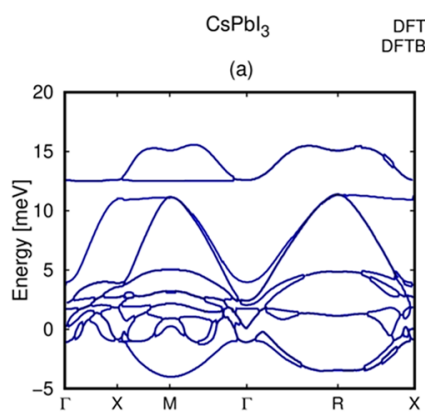

DFT $=\quad \mathrm{CsPbBr}_{3}$
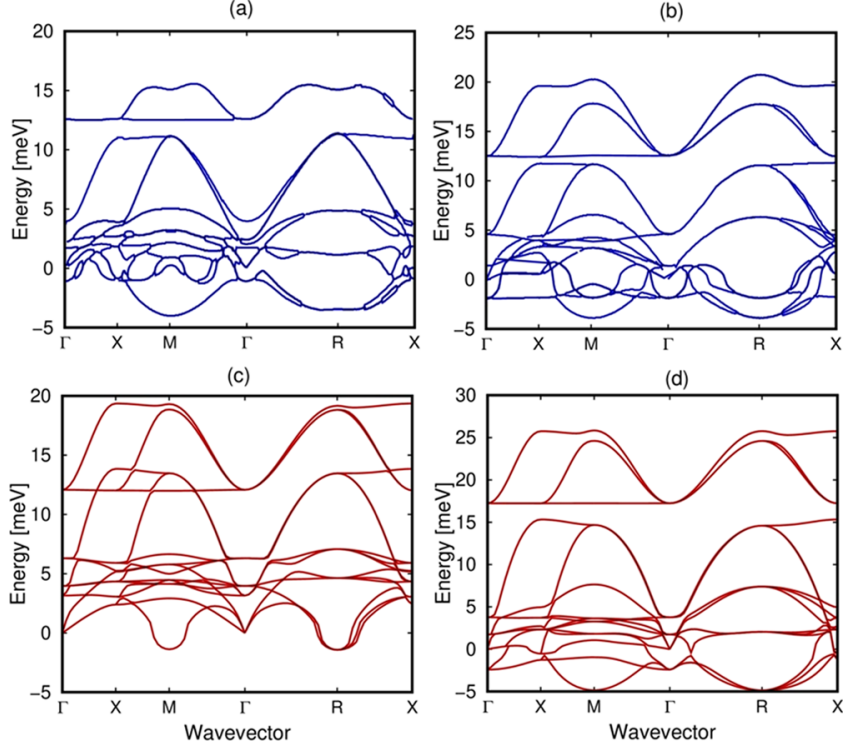

(d)

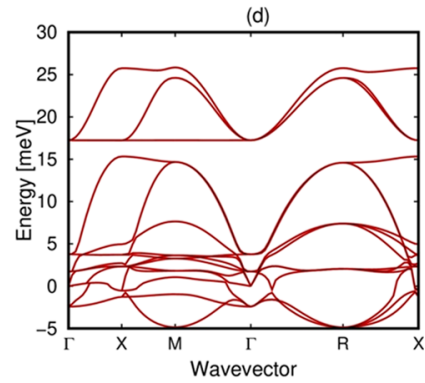

Figure 10. Phonon dispersion of the cubic $\mathrm{CsPbI}_{3}(\mathrm{a}, \mathrm{c})$ and $\mathrm{CsPbBr}$ (b,d) computed with GFN1-xTB (DFTB, red lines). Reference DFT data (blue lines) were taken from the literature. ${ }^{56,57}$

more stable orthorhombic phases (Figure S7), in agreement with the data reported in previous works. ${ }^{51}$

In addition to the Cs-based perovskites, we also found a similar satisfactory performance for MA-based MHPs. Figure S8 shows the phonon dispersion for the cubic and orthorhombic $\mathrm{MAPbI}_{3}$, where once more than the expected suppression of the imaginary modes in the most stable phase is observed. Specifically, the phonon dispersion of the cubic $\mathrm{MAPbI}_{3}$ exhibits negative modes at $\mathrm{M}$ and $\mathrm{R}$, while for the orthorhombic phase, the lowest phonon modes have zero frequency at the gamma point, in good agreement with the data reported by Walsh et al. ${ }^{19}$ These results suggest that GFN1-xTB is a valid tool for the efficient and accurate description of the vibrational properties of MHPs.

\section{CONCLUSIONS}

This work provides a comprehensive overview of the performance of the semiempirical GFN1-xTB tight binding method for the study of MHPs. Our analysis suggests that this method is suitable for the description of a variety of properties of the most common MHPs with reasonable accuracy. Such properties are (1) energetic and geometrical properties such as equations of state, rotation energy barriers of organic cations, and geometrical relaxation; (2) electronic properties such as band structures, band gaps, and partial DOS; and (3) vibrational properties such as phonon dispersions.

Despite its general effectiveness, GFN1-xTB has some shortcomings that do not yet allow for the accurate computation of specific material properties or particular chemical compositions. We identified two main limitations. The first one is the undesirable structural distortion of certain structures after a full geometry optimization. In this regard, orthorhombic phases can deviate up to $20-30 \%$ from the reference data, in contrast to the cubic crystals that show a maximum deviation lower than $10-15 \%$. The second limitation is the inaccurate description of the charged molecules with double or triple bonds, such as $\mathrm{FA}^{+}$cations, which extends to the description of their electronic behavior.

The tunability of GFN1-xTB nevertheless will potentially allow us to overcome the mentioned deficiencies. The GFN1xTB Hamiltonian contains various independent terms (electronic, repulsive, dispersion, and halogen-bonding terms) based on adjustable parameters that can be fitted to improve the quality of the results. Future works should focus on refining the repulsive potential parameters to avoid the observed deviation of interatomic distances and achieve the prediction of more accurate geometries. Modifying the electronic term parameters so that double and triple bonds in charged systems are properly accounted for is also necessary. With further work on this line, we believe that the GFN1-xTB method can become a powerful tool to simulate not only MHPs but also other systems in the area of materials science and beyond.

\section{DATA AND SOFTWARE AVAILABILITY}

Data concerning the results presented here, as well as input and output files, and DFT and GFN1-xTB-optimized MHPs are available from the corresponding author upon reasonable request and without restriction. The AMS2019.3 software used for GFN1-xTB calculations can be purchased at the https:// www.scm.com/support/downloads/ website, and VASP software version 5.4.1 to generate the reference DFT data can be purchased at the https://www.softwaresuggest.com/us/vasp website. An evaluation or demo license of both software packages is provided by the vendor upon request. All the figures were made with GNUPLOT 5.2 which is freely available from the http://www.gnuplot.info/download.html website.

\section{ASSOCIATED CONTENT}

\section{SI Supporting Information}

The Supporting Information is available free of charge at https://pubs.acs.org/doi/10.1021/acs.jcim.1c00432.

Additional equation of states, organic cation energy barriers, DOS, and phonon dispersion band structure (PDF)

\section{AUTHOR INFORMATION}

\section{Corresponding Author}

Shuxia Tao - Materials Simulation and Modelling, Department of Applied Physics and Center for Computational Energy Research, Department of Applied Physics, Eindhoven University of Technology, $5600 \mathrm{MB}$ Eindhoven, The Netherlands; 10 orcid.org/0000-00023658-8497; Email: s.x.tao@tue.nl

\section{Authors}

José Manuel Vicent-Luna - Materials Simulation and Modelling, Department of Applied Physics and Center for Computational Energy Research, Department of Applied Physics, Eindhoven University of Technology, $5600 \mathrm{MB}$ Eindhoven, The Netherlands; 10 orcid.org/0000-00018712-5591

Sofia Apergi - Materials Simulation and Modelling, Department of Applied Physics and Center for Computational Energy Research, Department of Applied Physics, Eindhoven University of Technology, 5600 MB 
Eindhoven, The Netherlands; $\odot$ orcid.org/0000-00016927-3478

Complete contact information is available at:

https://pubs.acs.org/10.1021/acs.jcim.1c00432

\section{Notes}

The authors declare no competing financial interest.

\section{ACKNOWLEDGMENTS}

J.M.V.L. and S.A. acknowledge funding support from NWO (Netherlands Organization for Scientific Research) STARTUP from the Netherlands. S.T. acknowledges funding by the Computational Sciences for Energy Research (CSER) tenure track program of Shell and NWO (project no. 15CST04-2) as well as NWO START-UP from the Netherlands. The authors also thank stimulating discussions within the SCALEUP consortium (SOLAR-ERA.NET Cofund 2, id: 32).

\section{REFERENCES}

(1) Kojima, A.; Teshima, K.; Shirai, Y.; Miyasaka, T. Organometal Halide Perovskites as Visible-Light Sensitizers for Photovoltaic Cells. J. Am. Chem. Soc. 2009, 131, 6050-6051.

(2) Jena, A. K.; Kulkarni, A.; Miyasaka, T. Halide Perovskite Photovoltaics: Background, Status, and Future Prospects. Chem. Rev. 2019, 119, 3036-3103.

(3) Kim, J. Y.; Lee, J.-W.; Jung, H. S.; Shin, H.; Park, N.-G. HighEfficiency Perovskite Solar Cells. Chem. Rev. 2020, 120, 7867-7918.

(4) Pacchioni, G. Highly Efficient Perovskite Leds. Nat. Nanotechnol. 2021, 6, 108.

(5) Tan, Z.-K.; Moghaddam, R. S.; Lai, M. L.; Docampo, P.; Higler, R.; Deschler, F.; Price, M.; Sadhanala, A.; Pazos, L. M.; Credgington, D.; Hanusch, F.; Bein, T.; Snaith, H. J.; Friend, R. H. Bright LightEmitting Diodes Based on Organometal Halide Perovskite. Nat. Nanotechnol. 2014, 9, 687-692.

(6) Ansari, M. I. H.; Qurashi, A.; Nazeeruddin, M. K. Frontiers, Opportunities, and Challenges in Perovskite Solar Cells: A Critical Review. J. Photochem. Photobiol., C 2018, 35, 1-24.

(7) Tonui, P.; Oseni, S. O.; Sharma, G.; Yan, Q.; Tessema Mola, G. Perovskites Photovoltaic Solar Cells: An Overview of Current Status. Renew. Sustain. Energy Rev. 2018, 91, 1025-1044.

(8) Niu, G.; Guo, X.; Wang, L. Review of Recent Progress in Chemical Stability of Perovskite Solar Cells. J. Mater. Chem. A 2015, 3, 8970-8980.

(9) Correa-Baena, J.-P.; Saliba, M.; Buonassisi, T.; Grätzel, M.; Abate, A.; Tress, W.; Hagfeldt, A. Promises and Challenges of Perovskite Solar Cells. Science 2017, 358, 739-744.

(10) Ünlü, F.; Jung, E.; Haddad, J.; Kulkarni, A.; Öz, S.; Choi, H.; Fischer, T.; Chakraborty, S.; Kirchartz, T.; Mathur, S. Understanding the Interplay of Stability and Efficiency in a-Site Engineered Lead Halide Perovskites. APL Mater. 2020, 8, 070901.

(11) Liu, G.; Xu, S.; Zheng, H.; Xu, X.; Xu, H.; Zhang, L.; Zhang, X.; Kong, F.; Pan, X. Boosting Photovoltaic Properties and Intrinsic Stability for Ma-Based Perovskite Solar Cells by Incorporating 1,1,1Trimethylhydrazinium Cation. ACS Appl. Mater. Interfaces 2019, 11, 38779-38788.

(12) Jiang, J.; Liu, F.; Tranca, I.; Shen, Q.; Tao, S. Atomistic and Electronic Origin of Phase Instability of Metal Halide Perovskites. ACS Appl. Energy Mater. 2020, 3, 11548-11558.

(13) Zhou, Y.; Xue, H.; Jia, Y. H.; Brocks, G.; Tao, S.; Zhao, N. Enhanced Incorporation of Guanidinium in Formamidinium-Based Perovskites for Efficient and Stable Photovoltaics: The Role of Cs and Br. Adv. Funct. Mater. 2019, 29, 1905739.

(14) Kwak, K.; Lim, E.; Ahn, N.; Heo, J.; Bang, K.; Kim, S. K.; Choi, M. An Atomistic Mechanism for the Degradation of Perovskite Solar Cells by Trapped Charge. Nanoscale 2019, 11, 11369-11378.

(15) Yu, C.-J. Advances in Modelling and Simulation of Halide Perovskites for Solar Cell Applications. JPhys Energy 2019, 1, 022001.
(16) Tao, S.; Schmidt, I.; Brocks, G.; Jiang, J.; Tranca, I.; Meerholz, K.; Olthof, S. Absolute Energy Level Positions in Tin- and Lead-Based Halide Perovskites. Nat. Commun. 2019, 10, 2560.

(17) Mosconi, E.; Amat, A.; Nazeeruddin, M. K.; Grätzel, M.; De Angelis, F. First-Principles Modeling of Mixed Halide Organometal Perovskites for Photovoltaic Applications. J. Phys. Chem. C 2013, 117, 13902-13913.

(18) Tao, S. X.; Cao, X.; Bobbert, P. A. Accurate and Efficient Band Gap Predictions of Metal Halide Perovskites Using the DFT-1/2 Method: GW Accuracy with DFT Expense. Sci. Rep. 2017, 7, 14386.

(19) Whalley, L. D.; Skelton, J. M.; Frost, J. M.; Walsh, A. Phonon Anharmonicity, Lifetimes, and Thermal Transport in $\mathrm{CH}_{3} \mathrm{NH}_{3} \mathrm{PbI}_{3}$ from Many-Body Perturbation Theory. Phys. Rev. B: Condens. Matter Mater. Phys. 2016, 94, 220301.

(20) Ciccioli, A.; Latini, A. Thermodynamics and the Intrinsic Stability of Lead Halide Perovskites $\mathrm{CH}_{3} \mathrm{NH}_{3} \mathrm{PbX}_{3}$. J. Phys. Chem. Lett. 2018, 9, 3756-3765.

(21) Meggiolaro, D.; Mosconi, E.; De Angelis, F. Modeling the Interaction of Molecular Iodine with $\mathrm{MAPbI}_{3}$ : A Probe of LeadHalide Perovskites Defect Chemistry. ACS Energy Lett. 2018, 3, 447451.

(22) Eames, C.; Frost, J. M.; Barnes, P. R. F.; O’Regan, B. C.; Walsh, A.; Islam, M. S. Ionic Transport in Hybrid Lead Iodide Perovskite Solar Cells. Nat. Commun. 2015, 6, 7497.

(23) Balestra, S. R. G.; Vicent-Luna, J. M.; Calero, S.; Tao, S.; Anta, J. A. Efficient Modelling of Ion Structure and Dynamics in Inorganic Metal Halide Perovskites. J. Mater. Chem. A 2020, 8, 11824-11836.

(24) Barboni, D.; De Souza, R. A. The Thermodynamics and Kinetics of Iodine Vacancies in the Hybrid Perovskite Methylammonium Lead Iodide. Energy Environ. Sci. 2018, 11, 3266-3274.

(25) Mattoni, A.; Filippetti, A.; Saba, M. I.; Delugas, P. Methylammonium Rotational Dynamics in Lead Halide Perovskite by Classical Molecular Dynamics: The Role of Temperature. J. Phys. Chem. C 2015, 119, 17421-17428.

(26) Gehrmann, C.; Egger, D. A. Dynamic Shortening of Disorder Potentials in Anharmonic Halide Perovskites. Nat. Commun. 2019, 10, 3141.

(27) Zhou, Y.; Zhou, Z.; Chen, M.; Zong, Y.; Huang, J.; Pang, S.; Padture, N. P. Doping and Alloying for Improved Perovskite Solar Cells. J. Mater. Chem. A 2016, 4, 17623-17635.

(28) Chen, Y.; Zhou, H. Defects Chemistry in High-Efficiency and Stable Perovskite Solar Cells. J. Appl. Phys. 2020, 128, 060903.

(29) Xu, L.; Chen, J.; Song, J.; Li, J.; Xue, J.; Dong, Y.; Cai, B.; Shan, Q.; Han, B.; Zeng, H. Double-Protected All-Inorganic Perovskite Nanocrystals by Crystalline Matrix and Silica for Triple-Modal AntiCounterfeiting Codes. ACS Appl. Mater. Interfaces 2017, 9, 2655626564.

(30) Yadav, S. K.; Grandhi, G. K.; Dubal, D. P.; Mello, J. C.; Zbořil, R.; Fischer, R. A.; Jayaramulu, K.; Jayaramulu, K. Metal Halide Perovskite@Metal-Organic Framework Hybrids: Synthesis, Design, Properties, and Applications. Small 2020, 16, 2004891.

(31) English, N. J. Dynamical Properties of Organo Lead-Halide Perovskites and Their Interfaces to Titania: Insights from Density Functional Theory. Heliyon 2020, 6, No. e03427.

(32) Apergi, S.; Brocks, G.; Tao, S. Tuning the Electronic Levels of Nio with Alkali Halides Surface Modifiers for Perovskite Solar Cells. Phys. Rev. Mater. 2020, 4, 085403.

(33) Christensen, A. S.; Kubař, T.; Cui, Q.; Elstner, M. Semiempirical Quantum Mechanical Methods for Noncovalent Interactions for Chemical and Biochemical Applications. Chem. Rev. 2016, 116, 5301-5337.

(34) Elstner, M.; Porezag, D.; Jungnickel, G.; Elsner, J.; Haugk, M.; Frauenheim, T.; Suhai, S.; Seifert, G. Self-Consistent-Charge DensityFunctional Tight-Binding Method for Simulations of Complex Materials Properties. Phys. Rev. B: Condens. Matter Mater. Phys. 1998, 58, 7260-7268.

(35) Grimme, S.; Bannwarth, C.; Shushkov, P. A Robust and Accurate Tight-Binding Quantum Chemical Method for Structures, Vibrational Frequencies, and Noncovalent Interactions of Large 
Molecular Systems Parametrized for All Spd-Block Elements ( $\mathrm{Z}=1-$ 86). J. Chem. Theory Comput. 2017, 13, 1989-2009.

(36) SCM. Ams2019, Theoretical Chemistry; Vrije Universiteit: Amsterdam, The Netherlands, 2019. http://www.scm.com.

(37) Bannwarth, C.; Caldeweyher, E.; Ehlert, S.; Hansen, A.; Pracht, P.; Seibert, J.; Spicher, S.; Grimme, S. Extended Tight-Binding Quantum Chemistry Methods. Wiley Interdiscip. Rev.: Comput. Mol. Sci. 2021, 11, No. e1493.

(38) Grimme, S.; Antony, J.; Ehrlich, S.; Krieg, H. A Consistent and Accurate Ab Initio Parametrization of Density Functional Dispersion Correction (DFT-D) for the 94 Elements H-Pu. J. Chem. Phys. 2010, 132, 154104.

(39) Johnson, E. R.; Becke, A. D. A Post-Hartree-Fock Model of Intermolecular Interactions. J. Chem. Phys. 2005, 123, 024101.

(40) Bitzek, E.; Koskinen, P.; Gähler, F.; Moseler, M.; Gumbsch, P. Structural Relaxation Made Simple. Phys. Rev. Lett. 2006, 97, 170201.

(41) Blöchl, P. E. Projector Augmented-Wave Method. Phys. Rev. B: Condens. Matter Mater. Phys. 1994, 50, 17953-17979.

(42) Kresse, G.; Hafner, J. Ab Initio Molecular Dynamics for Liquid Metals. Phys. Rev. B: Condens. Matter Mater. Phys. 1993, 47, 558-561.

(43) Kresse, G.; Joubert, D. From Ultrasoft Pseudopotentials to the Projector Augmented-Wave Method. Phys. Rev. B: Condens. Matter Mater. Phys. 1999, 59, 1758-1775.

(44) Kresse, G.; Furthmüller, J. Efficient Iterative Schemes for Ab Initio Total-Energy Calculations Using a Plane-Wave Basis Set. Phys. Rev. B: Condens. Matter Mater. Phys. 1996, 54, 11169-11186.

(45) Perdew, J. P.; Burke, K.; Ernzerhof, M. Generalized Gradient Approximation Made Simple. Phys. Rev. Lett. 1996, 77, 3865-3868.

(46) Civalleri, B.; Zicovich-Wilson, C. M.; Valenzano, L.; Ugliengo, P. B3LYP Augmented with an Empirical Dispersion Term (B3LYP$\mathrm{D}^{*}$ ) as Applied to Molecular Crystals. Crystengcomm 2008, 10, 405410 .

(47) Quertinmont, J.; Carletta, A.; Tumanov, N. A.; Leyssens, T.; Wouters, J.; Champagne, B. Assessing Density Functional Theory Approaches for Predicting the Structure and Relative Energy of Salicylideneaniline Molecular Switches in the Solid State. J. Phys. Chem. C 2017, 121, 6898-6908.

(48) Quertinmont, J.; Leyssens, T.; Wouters, J.; Champagne, B. Effects of Empirical Dispersion Energy on the Geometrical Parameters and Relative Energy of a Salicylideneaniline Molecular Switch in the Solid State. Crystals 2018, 8, 125.

(49) Ruggiero, M. T.; Gooch, J.; Zubieta, J.; Korter, T. M. Evaluation of Range-Corrected Density Functionals for the Simulation of Pyridinium-Containing Molecular Crystals. J. Phys. Chem. A. 2016, 120, 939-947.

(50) Cutini, M.; Maschio, L.; Ugliengo, P. Exfoliation Energy of Layered Materials by DFT-D: Beware of Dispersion! J. Chem. Theory Comput. 2020, 16, 5244-5252.

(51) Marronnier, A.; Roma, G.; Boyer-Richard, S.; Pedesseau, L.; Jancu, J.-M.; Bonnassieux, Y.; Katan, C.; Stoumpos, C. C.; Kanatzidis, M. G.; Even, J. Anharmonicity and Disorder in the Black Phases of Cesium Lead Iodide Used for Stable Inorganic Perovskite Solar Cells. ACS Nano 2018, 12, 3477-3486.

(52) Brivio, F.; Frost, J. M.; Skelton, J. M.; Jackson, A. J.; Weber, O. J.; Weller, M. T.; Goñi, A. R.; Leguy, A. M. A.; Barnes, P. R. F.; Walsh, A. Lattice Dynamics and Vibrational Spectra of the Orthorhombic, Tetragonal, and Cubic Phases of Methylammonium Lead Iodide. Phys. Rev. B: Condens. Matter Mater. Phys. 2015, 92, 144308.

(53) Rodová, M.; Brožek, J.; Knížek, K.; Nitsch, K. Phase Transitions in Ternary Caesium Lead Bromide. J. Therm. Anal. Calorim. 2003, 71, 667-673.

(54) Su, J.; Chen, D. P.; Lin, C. T. Growth of Large $\mathrm{CH}_{3} \mathrm{NH}_{3} \mathrm{PbX}$ (X=I, Br) Single Crystals in Solution. J. Cryst. Growth 2015, 422, 7579.

(55) Nazarenko, O.; Yakunin, S.; Morad, V.; Cherniukh, I.; Kovalenko, M. V. Single Crystals of Caesium Formamidinium Lead Halide Perovskites: Solution Growth and Gamma Dosimetry. NPG Asia Mater. 2017, 9, No. e373.
(56) Myung, C. W.; Yun, J.; Lee, G.; Kim, K. S. A New Perspective on the Role of a-Site Cations in Perovskite Solar Cells. Adv. Energy Mater. 2018, 8, 1702898.

(57) Guo, P.; Xia, Y.; Gong, J.; Stoumpos, C. C.; McCall, K. M.; Alexander, G. C. B.; Ma, Z.; Zhou, H.; Gosztola, D. J.; Ketterson, J. B.; Kanatzidis, M. G.; Xu, T.; Chan, M. K. Y.; Schaller, R. D. Polar Fluctuations in Metal Halide Perovskites Uncovered by Acoustic Phonon Anomalies. ACS Energy Lett. 2017, 2, 2463-2469. 